\title{
Conference Paper \\ Geometric Hamiltonian Formulation of a Variational Problem Depending on the Covariant Acceleration
}

\author{
Lígia Abrunheiro, ${ }^{1,2}$ Margarida Camarinha, ${ }^{3}$ and Jesús Clemente-Gallardo ${ }^{4}$ \\ ${ }^{1}$ Center for Research and Development in Mathematics and Applications (CIDMA), Department of Mathematics, \\ University of Aveiro, 3810-193 Aveiro, Portugal \\ ${ }^{2}$ ISCA, University of Aveiro, 3810-500 Aveiro, Portugal \\ ${ }^{3}$ CMUC, Department of Mathematics, University of Coimbra, 3001-501 Coimbra, Portugal \\ ${ }^{4}$ BIFI, Department of Theoretical Physics and Unidad Asociada IQFR-BIFI, University of Zaragoza, Edificio I+D, \\ Campus Río Ebro, C/ Mariano Esquillor s/n, 50018 Zaragoza, Spain \\ Correspondence should be addressed to Lígia Abrunheiro; abrunheiroligia@ua.pt
}

Received 15 June 2013; Accepted 14 July 2013

Academic Editors: G. S. F. Frederico, N. Martins, and D. F. M. Torres

This Conference Paper is based on a presentation given by Lígia Abrunheiro at "The Cape Verde International Days on Mathematics 2013" held from 22 April 2013 to 25 April 2013 in Praia, Cape Verde.

Copyright ( $) 2013$ Lígia Abrunheiro et al. This is an open access article distributed under the Creative Commons Attribution License, which permits unrestricted use, distribution, and reproduction in any medium, provided the original work is properly cited.

We consider a second-order variational problem depending on the covariant acceleration, which is related to the notion of Riemannian cubic polynomials. This problem and the corresponding optimal control problem are described in the context of higher order tangent bundles using geometric tools. The main tool, a presymplectic variant of Pontryagin's maximum principle, allows us to study the dynamics of the control problem.

\section{Introduction}

In [1], Skinner and Rusk obtained a unified formalism for Lagrangian and Hamiltonian dynamics of autonomous mechanical systems, and this issue has been extended in many directions. In particular, there is an increasing interest in the study of optimal control problems from that geometric viewpoint, which involves the presymplectic algorithm of Gotay-Nester [2].

Riemannian cubic polynomials can be seen as a generalization of cubic polynomials to non-Euclidean spaces $[3,4]$. These objects are stationary curves in a Riemannian manifold for a second-order variational problem with Lagrangian given by the norm squared covariant acceleration. There are many applications that inspire the study of those curves, namely, problems of interpolation in computer graphics and problems in the context of robotics and aeronautics as the trajectory planning of a rigid body.

As far as we know, the first Hamiltonian description of the optimal control problem whose control system is associated with the variational problem mentioned in the previous paragraph was considered in [5] but from a non-geometric perspective. The aim of this paper is to give a precise and geometric description of that optimal control problem. For this purpose, we adapt the presymplectic geometric version of the Pontryagin maximum principle based on the Skinner-Rusk methodology, which was proposed for the control theory by several authors (see, e.g., [6-9] and the references mentioned in these papers). Here, we develop the work started in $[10$, 11] where that intrinsic version of the problem was first presented. We specify to our problem all the details of the presymplectic approach and reduce the study of the problem to the study of an interesting symplectic Hamiltonian system.

The approach used in this work has important implications from the point of view of the integrability of the dynamical system on compact and connected Lie groups. For a detailed description of the optimal control problem for compact and connected Lie groups, we refer the interested reader to [12-14]. 
The organization of the paper is as follows. In Section 2, we review the concept and some properties of higher order tangent bundles. We also recall the geometric formulation of optimal control problems and its adaptation to SkinnerRusk methodology. Section 3 contains the geometric formulation of the second-order variational problem whose EulerLagrange equation is the fourth-order differential equation that defines the cubic polynomial curves on Riemannian manifolds. Section 4 is devoted to the main results of this paper; we consider the second-order optimal control problem corresponding to the variational problem presented in Section 3 and use the presymplectic constraint algorithm to describe its dynamics. In the last section, some examples are provided in order to illustrate these geometric tools.

\section{Preliminary Results}

Consider a differentiable manifold $M$ of finite dimension $n$. Let $\left(x^{1}, \ldots, x^{n}\right)$ be a local coordinate system on $M$, simply denoted by $\left(x^{i}\right)$. In the paper, we assume similar simplifications to coordinate notations.

2.1. Higher Order Tangent Bundles. The tangent bundle of $M$ can be seen as a trivial example of higher order tangent bundles. We recall very briefly some basic tools from the geometry of those bundles. For further details, see [15].

Consider the following well-defined equivalence relation on the set of smooth curves in $M$.

We say that two smooth curves in $M, \gamma_{1}$, and $\gamma_{2}$, defined on an interval $(-a, a)$ with $a \in \mathbb{R}$, have contact of order $k$ at 0 if $\gamma_{1}(0)=\gamma_{2}(0)=x$ and for a local coordinate system $(U, \varphi)$ on $M$ around $x$, the derivatives of $\varphi \circ \gamma_{1}$ and $\varphi \circ \gamma_{2}$ up to order $k$, included, coincide at 0 .

The equivalence class determined by a curve $\gamma$ is denoted by $[\gamma]_{0}^{k}$ and is called a $k$-jet or $k$-velocity. The tangent bundle of order $k$ of $M$, represented by $T^{k} M$, is defined as being the set of all equivalence classes. The tangent bundle $T^{k} M$ is a $(k+$ 1) $n$-dimensional manifold, and it is also a fibered manifold over $M$ with projection

$$
\pi_{k}: T^{k} M \longrightarrow M, \quad[\gamma]_{0}^{k} \longmapsto \gamma(0)=x .
$$

A system $\left(U, x^{i}\right)$ of local coordinates on $M$ induces natural local coordinates on $T^{k} M$ given by $\left(\pi_{k}^{-1}(U), x_{0}^{i} ; x_{1}^{i} ; x_{2}^{i} ; \ldots\right.$; $\left.x_{k}^{i}\right)$, where

$$
x_{l}^{i}: \pi_{k}^{-1}(U) \subset T^{k} M \longrightarrow \mathbb{R},\left.\quad[\gamma]_{0}^{k} \longmapsto \frac{d^{l}}{d t^{l}}\left(x^{i} \circ \gamma\right)(t)\right|_{t=0},
$$

for $l=0, \ldots, k$ and $i=1, \ldots, n$. If $k=0$, the tangent bundle $T^{0} M$ is identified with the manifold $M$ and for $k=1, T^{1} M$ is just the tangent bundle of $M, T M$.

We have the canonical projections

$$
\tau_{k}^{l}: T^{k} M \longrightarrow T^{l} M, \quad[\gamma]_{0}^{k} \longmapsto[\gamma]_{0}^{l}, \quad l=0, \ldots, k
$$

which define several different fibered structures on $T^{k} M$. Note that $\tau_{k}^{0}=\pi_{k}$. Locally, $\tau_{k}^{l}\left(x_{0}^{i} ; x_{1}^{i} ; x_{2}^{i} ; \ldots ; x_{k}^{i}\right)=$ $\left(x_{0}^{i} ; x_{1}^{i} ; x_{2}^{i} ; \ldots ; x_{l}^{i}\right)$.

Given a smooth curve $\gamma$ in $M$, the lift to $T^{k} M$ of $\gamma$ is a smooth curve in $T^{k} M$ defined by $\gamma_{k}(t)=\left[\gamma_{t}\right]_{0}^{k}$, where $\gamma_{t}(s)=\gamma(t+s)$. If $\gamma$ is given locally by $\left(x^{i}\right)$, then $\gamma_{k}$ is locally represented by $\left(x^{i} ; d x^{i} / d t ; \ldots ; d^{k} x^{i} / d t^{k}\right)$.

We can also consider natural injections $i_{k, l}: T^{k+l} M \rightarrow$ $T^{k}\left(T^{l} M\right),[\gamma]_{0}^{k+l} \mapsto\left[\gamma_{l}\right]_{0}^{k}$, for $l=1, \ldots, k$.

Here, we are particularly interested in the secondorder tangent bundle $T^{2} M$. We denote the canonical local coordinates on $T M$ and $T^{2} M$ by $\left(x^{1}, \ldots, x^{n} ; y^{1}, \ldots, y^{n}\right)=$ $\left(x^{i} ; y^{i}\right)$ and $\left(x^{1}, \ldots, x^{n} ; y^{1}, \ldots, y^{n} ; u^{1}, \ldots, u^{n}\right)=\left(x^{i} ; y^{i} ; u^{i}\right)$, respectively. The natural bases of the tangent spaces $T_{x} M$, $T_{(x, y)} T M$, and $T_{(x, y, u)} T^{2} M$ are denoted, respectively, by $\left\{\partial / \partial x^{i}\right\},\left\{\partial / \partial x^{i}, \partial / \partial y^{i}\right\}$, and $\left\{\partial / \partial x^{i}, \partial / \partial y^{i}, \partial / \partial u^{i}\right\}$, for $x \in M$, $(x, y) \in T M$, and $(x, y, u) \in T^{2} M$. For clarity, to distinguish the projections $\pi_{1}$ of the tangent bundles TM and TTM, we use the notations $\pi_{M}: T M \rightarrow M$ and $\pi_{T M}: T T M \rightarrow T M$. Moreover, the natural injection $i_{1,1}: T^{2} M \rightarrow T T M$ is locally defined by

$$
i_{1,1}\left(x^{i} ; y^{i} ; u^{i}\right)=\sum_{i=1}^{n}\left(y^{i} \frac{\partial}{\partial x^{i}}+u^{i} \frac{\partial}{\partial y^{i}}\right) .
$$

2.2. Geometric Description of an Optimal Control Problem. Let $B$ be a fiber bundle over $M$ with projection $\tau: B \rightarrow M$. Consider also a vector field $\Pi$ along the projection $\tau$; that is, a smooth map $\Pi: B \rightarrow$ TM such that the diagram

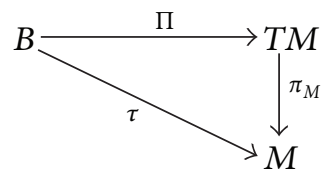

is commutative, where $\pi_{M}$ represents the natural canonical projection from $T M$ to $M$.

An optimal control problem with state space $M$ and control bundle $B$ consists in finding the curves $\alpha:[0, T] \rightarrow$ $B$ of class $C^{2}$, piecewise smooth and with $T \in \mathbb{R}^{+}$, with fixed initial and final conditions in the state space, satisfying

$$
\frac{d}{d t}(\tau \circ \alpha)=\Pi \circ \alpha
$$

and minimizing an integral functional $\int_{0}^{T} L(\alpha(t)) d t$, where $L$ is a smooth function $L: B \rightarrow \mathbb{R}$ called cost function.

Equation (6) is known as the control system, while an integral curve of $\Pi$, that is, a curve $\alpha$ in $B$ satisfying the control system, is called a trajectory of the control system. Note that if $\left(x^{i}\right)$ is a local coordinate system on $M$ and $\left(x^{i} ; u^{a}\right)$ are natural coordinates on $B(i=1, \ldots, n, a=1, \ldots, m$, with $n+m=\operatorname{dim} B)$, then the control system is characterized by the system of differential equations

$$
\dot{x}^{i}=\Pi^{i}(x, u), \quad i=1, \ldots, n,
$$


for $(x, u) \in B$ and where $\Pi^{i}$ represent the canonical local coordinates of $\Pi$ on $T M$, that is, $\Pi(x, u)=\sum_{i=1}^{n}$ $\left.\Pi^{i}(x, u)\left(\partial / \partial x^{i}\right)\right|_{\tau(x, u)}$.

The costate space of the system is the cotangent bundle $T^{*} M$ with natural canonical projection $\tau_{M}: T^{*} M \rightarrow M$. The dynamics of the control system can be described by a symplectic or a presymplectic Hamiltonian system (see, e.g., [6-9]). Here, we are interested in the presymplectic description, and hence we should consider the presymplectic Hamiltonian system $(\mathscr{T}, \Omega, H)$ with the following.

(i) The total space $\mathscr{T}=T^{*} M \times_{M} B$ a fiber bundle over the manifold $M$ with canonical projections $p r_{1}$ : $T^{*} M \times_{M} B \rightarrow T^{*} M$ and $p r_{2}: T^{*} M \times_{M} B \rightarrow B$.

(ii) The canonical presymplectic form $\Omega$ on $\mathscr{T}$ (i.e., a closed 2 -form which may be degenerate) given by the pullback of the canonical symplectic form $\omega_{0}$ on $T^{*} M$ (i.e., a closed and nondegenerate 2 -form) by the projection $p r_{1}, \Omega=\left(p r_{1}\right)^{*} \omega_{0}$. Locally,

$$
\Omega=\sum_{i=1}^{n} d x^{i} \wedge d p_{i}
$$

where $\left(x^{i} ; p_{i}\right)$ are the natural local coordinates on $T^{*} M$ induced by $\left(x^{i}\right)$. Note that the kernel of $\Omega$ is locally given by $\partial / \partial u^{a}=0$.

(iii) The Hamiltonian $H: T^{*} M \times_{M} B \rightarrow \mathbb{R}$ defined by $H=\left\langle\left\langle p r_{1}, \Pi \circ p r_{2}\right\rangle\right\rangle-L \circ p r_{2}$, where $\langle\langle\cdot, \cdot\rangle\rangle$ represents the pairing duality of vectors and covectors on $M$. We get

$$
H(x, p, u)=\sum_{i=1}^{n} p_{i} \Pi^{i}(x, u)-L(x, u),
$$

for each $(x, p, u) \in \mathscr{T}$ (i.e., $x \in M, p \in T_{x}^{*} M$ and $(x, u) \in B$, with $\tau(x, u)=x)$.

The dynamics of the presymplectic Hamiltonian system $(\mathscr{T}, \Omega, H)$ is determined by the vector field $X_{H}$ solution of the equation

$$
i_{X_{H}} \Omega=d H .
$$

Equation (10) is interpreted as an intrinsic version of the Hamiltonian equations that come from the maximum principle of Pontryagin in the sense that a curve $\alpha$ in $B$ is a trajectory of the optimal control problem if there exists a lifting of $\alpha$ to the total space $\mathscr{T}$ which is an integral curve of the vector field $X_{H}$. Notice the following.

(i) Locally, $X_{H}=\sum_{i=1}^{n}\left(A^{i}\left(\partial / \partial x^{i}\right)+B_{i}\left(\partial / \partial p_{i}\right)\right)+$ $\sum_{a=1}^{m} C^{a}\left(\partial / \partial u^{a}\right)$, where $A^{i}, B_{i}$, and $C^{a}$ are smooth functions on $\mathscr{T}$. Hence, $i_{X_{H}} \Omega=\sum_{i=1}^{n}\left(A^{i} d p_{i}-B_{i} d x^{i}\right)$.

(ii) On the other hand, $d H=\sum_{i=1}^{n}\left(\left(\partial H / \partial x^{i}\right) d x^{i}+\right.$ $\left.\left(\partial H / \partial p_{i}\right) d p_{i}\right)+\sum_{a=1}^{m}\left(\partial H / \partial u^{a}\right) d u^{a}$.

(iii) So, (10) is equivalent to $A^{i}=\partial H / \partial p_{i}, B_{i}=-\partial H / \partial x^{i}$, and $\partial H / \partial u^{a}=0, i=1, \ldots, n, a=1, \ldots, m$. (iv) Therefore, an integral curve of $X_{H}$, locally given by $\left(x^{i} ; p^{i} ; u^{i}\right)$, is such that

$$
\begin{array}{r}
\dot{x}^{i}=\frac{\partial H}{\partial p_{i}}, \quad \dot{p}_{i}=-\frac{\partial H}{\partial x^{i}}, \quad \frac{\partial H}{\partial u^{a}}=0, \\
i=1, \ldots, n, \quad a=1, \ldots, m
\end{array}
$$

That is, $X_{H}$ is the vector field

$$
X_{H}=\sum_{i=1}^{n}\left(\frac{\partial H}{\partial p_{i}} \frac{\partial}{\partial x^{i}}-\frac{\partial H}{\partial x^{i}} \frac{\partial}{\partial p_{i}}\right)
$$

defined in the subset $W_{1}=\left\{(x, p, u) \in \mathscr{T}: \partial H / \partial u^{a}=\right.$ $0, a=1, \ldots, m\}$.

In the geometric framework, we have

$$
W_{1}=\left\{z \in \mathscr{T}: d H(z)\left(v_{z}\right)=0, \forall v_{z} \in \operatorname{Ker} \Omega(z)\right\},
$$

where $\operatorname{Ker} \Omega(z)=\left\{v_{z} \in T_{z} \mathscr{T}: \Omega(z)\left(v_{z}, \cdot\right)=0\right\}$. Indeed, since $\Omega$ is presymplectic, we have to consider the points of $\mathscr{T}$ where (10) has solution. We assume that $W_{1}$ is a submanifold of $\mathscr{T}$. The dynamical vector field $X_{H}$ is determined by $\left.\left(i_{X_{H}} \Omega-d H\right)\right|_{W_{1}}=0$. However, the solution of that equation is not necessarily unique, and it is possible that there exist points on $W_{1}$ where the solution vector field is not tangent to $W_{1}$, and thus it does not necessarily induce a dynamics on $W_{1}$. If it is the case, we construct a second constraint submanifold $W_{2}$, that we assume to be a submanifold of $W_{1}$, defined by the points on $W_{1}$, where such a solution exists. But, again, it may happen, that we cannot guarantee the existence of a dynamics on $W_{2}$, and so the process may have to continue. This procedure is called the presymplectic constraint algorithm of Gotay-Nester [2]. The idea of the algorithm is to construct a chain of constraint manifolds until we find (if it exists) a final submanifold $W_{f}$, where exists at least one vector field tangent to that submanifold and satisfying the dynamical equation. If the optimal control problem is regular, then $W_{f}=W_{1}$.

\section{Second-Order Variational Problem}

From this section onwards, $M$ is a Riemannian manifold with Riemannian metric $\langle\cdot, \cdot\rangle$. We denote the symmetric connection on $M$ compatible with this metric by $\nabla$ and the corresponding covariant derivative along a curve in $M$ by $D X / d t$, where $X$ is a vector field along the curve. Moreover, we denote the curvature tensor field by $R$.

We are interested in the following second-order variational problem: find the curves that minimize

$$
J(\gamma)=\frac{1}{2} \int_{0}^{T}\left\langle\frac{D^{2} \gamma}{d t^{2}}, \frac{D^{2} \gamma}{d t^{2}}\right\rangle d t,
$$

over the class $\mathscr{C}$ of smooth curves $\gamma:[0, T] \rightarrow M$ satisfying the boundary conditions

$$
\begin{array}{ccc}
\gamma(0)=x_{0}, & & \gamma(T)=x_{T}, \\
\frac{d \gamma}{d t}(0)=y_{0}, & & \frac{d \gamma}{d t}(T)=y_{T},
\end{array}
$$


where $x_{0}, x_{T} \in M, y_{0} \in T_{x_{0}} M, y_{T} \in T_{x_{T}} M$, and $T \in$ $\mathbb{R}^{+}$. This problem was studied in 1989 by Noakes et al. on compact Lie groups [4] and later, in 1995, by Crouch and Silva Leite in the context of dynamic interpolation [3]. The Euler-Lagrange equation of the problem is the fourth-order differential equation

$$
\frac{D^{4} \gamma}{d t^{4}}+R\left(\frac{D^{2} \gamma}{d t^{2}}, \frac{d \gamma}{d t}\right) \frac{d \gamma}{d t}=0 .
$$

The solutions of this equation are called cubic polynomials on $M$.

Recall that if a curve $\gamma$ in $M$ is locally represented by $\left(x^{i}\right)$, then the velocity vector field along $\gamma$ is $d \gamma / d t=$ $\left.\sum_{i=1}^{n} \dot{x}^{i}\left(\partial / \partial x^{i}\right)\right|_{\gamma(t)}$ and the covariant acceleration of $\gamma$ is given by

$$
\frac{D^{2} \gamma}{d t^{2}}=\left.\sum_{k=1}^{n}\left(\ddot{x}^{k}+\sum_{i, j=1}^{n} \Gamma_{i j}^{k} \dot{x}^{i} \dot{x}^{j}\right) \frac{\partial}{\partial x^{k}}\right|_{\gamma(t)} .
$$

Here, $\Gamma_{i j}^{k}$ are the Christoffel symbols defining the Riemannian connection, which can be obtained using the identity

$$
\Gamma_{i j}^{k}=\frac{1}{2} \sum_{l=1}^{n} g^{k l}\left(\frac{\partial g_{j l}}{\partial x^{i}}+\frac{\partial g_{l i}}{\partial x^{j}}-\frac{\partial g_{i j}}{\partial x^{l}}\right),
$$

where $g_{i j}$ are the components of the Riemannian metric and $\left[g^{i j}\right]_{1 \leq i, j \leq n}$ is the inverse matrix of the matrix $\left[g_{i j}\right]_{1 \leq i, j \leq n}$. Moreover, the lift $\gamma_{2}$ to $T^{2} M$ of the curve $\gamma$ is locally represented by $\left(x^{i} ; \dot{x}^{i} ; \ddot{x}^{i}\right)$. Therefore, the action functional $J: \mathscr{C} \rightarrow \mathbb{R}$ of our problem can be written as $J(\gamma)=$ $\int_{0}^{T} L\left(\gamma_{2}(t)\right) d t$, where $L: T^{2} M \rightarrow \mathbb{R}$ is the Lagrangian of the problem. Locally, we have

$$
L\left(x^{i} ; y^{i} ; u^{i}\right)=\frac{1}{2} \sum_{i=1}^{n} g_{i j} \bar{u}^{i} \bar{u}^{j},
$$

with $\bar{u}^{k}=u^{k}+\sum_{i, j=1}^{n} \Gamma_{i j}^{k} y^{i} y^{j}$

Observe that the Lagrangian $L: T^{2} M \rightarrow \mathbb{R}$ of the problem is defined, for each $[\gamma]_{0}^{2} \in T^{2} M$, by

$$
L\left([\gamma]_{0}^{2}\right)=\frac{1}{2}\left\langle\left(K \circ i_{1,1}\right)\left([\gamma]_{0}^{2}\right),\left(K \circ i_{1,1}\right)\left([\gamma]_{0}^{2}\right)\right\rangle,
$$

for $i_{1,1}: T^{2} M \rightarrow T T M$ the natural injection defined by (4) and $K: T T M \rightarrow T M$ the connection application locally given by

$$
\begin{aligned}
& K\left(\sum_{i=1}^{n} X_{i} \frac{\partial}{\partial x^{i}}+\sum_{i=1}^{n} X_{i+n} \frac{\partial}{\partial y^{i}}\right) \\
& =\sum_{i=1}^{n}\left(X_{n+i}+\sum_{j, k=1}^{n} \Gamma_{j k}^{i} y^{j} X_{k}\right) \frac{\partial}{\partial x^{i}} .
\end{aligned}
$$

The Lagrangian $L$ defines a dynamics on the third-order tangent space $T^{3} M$ since the Euler-Lagrange equation (16) can be interpreted as a vector field on $T^{3} M$, whose integral curves are lifts to $T^{3} M$ of curves in $M$ solutions of the EulerLagrange equation.

\section{Second-Order Optimal Control Problem}

The control system associated with the variational problem of the previous section is a control system of second-order on $M$. We now adapt for that situation the geometric description of Section 2.2.

4.1. Geometric Formulation of the Optimal Control Problem. The second-order control system on $M$ that we are interested in is

$$
\frac{D^{2} \gamma}{d t^{2}}=\left.\sum_{i=1}^{n} u^{i} \frac{\partial}{\partial x^{i}}\right|_{\gamma(t)}
$$

where $\gamma:[0, T] \rightarrow M$ is a curve in $M$ and $u^{i}$ are real smooth functions called control functions. If $\gamma$ is locally represented by $\left(x^{i}\right)$, then the control system is written as the set of differential equations dependent of the parameters $u^{i}$

$$
\ddot{x}^{i}=u^{i}-\sum_{j, k=1}^{n} \Gamma_{j k}^{i} \dot{x}^{j} \dot{x}^{k}, \quad i=1, \ldots, n,
$$

since $D^{2} \gamma / d t^{2}$ is given by (17). Note that the system is affine on the controls.

From a geometric point of view, the control system can be described by a vector field $\Pi$ along the natural projection $\tau_{2}^{1}: T^{2} M \rightarrow T M$, that is, a smooth map $\Pi: T^{2} M \rightarrow T T M$ such that the following diagram is commutative:

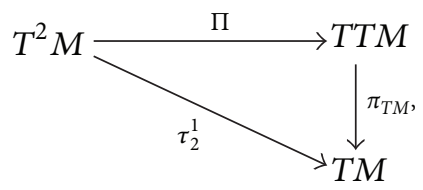

where $\pi_{T M}$ is the canonical projection. The state space is $T M$ and $T^{2} M$ is the control bundle. If $[\gamma]_{0}^{2} \in T^{2} M$, we know that $\tau_{2}^{1}\left([\gamma]_{0}^{2}\right)=[\gamma]_{0}^{1} \in T M$ and hence $\Pi\left([\gamma]_{0}^{2}\right) \in T_{[\gamma]_{0}^{1}} T M$. Consequently, for real smooth functions $\Pi^{i}: T^{2} M \rightarrow \mathbb{R}$, with $i=1, \ldots, n$, the vector field $\Pi$ can be expressed as $\Pi\left([\gamma]_{0}^{2}\right)=$ $\left.\sum_{i=1}^{n} \Pi^{i}\left([\gamma]_{0}^{2}\right)\left(\partial / \partial x^{i}\right)\right|_{[\gamma]_{0}^{1}}+\left.\sum_{i=1}^{n} \Pi^{i+n}\left([\gamma]_{0}^{2}\right)\left(\partial / \partial y^{i}\right)\right|_{[\gamma]_{0}^{1}}$. Along a curve $\alpha:[0, T] \rightarrow T^{2} M$, we have

$$
\Pi \circ \alpha=\left.\sum_{i=1}^{n}\left(\Pi^{i} \circ \alpha\right) \frac{\partial}{\partial x^{i}}\right|_{\left(\tau_{2}^{1} \circ \alpha\right)(t)}+\left.\sum_{i=1}^{n}\left(\Pi^{i+n} \circ \alpha\right) \frac{\partial}{\partial y^{i}}\right|_{\left(\tau_{2}^{1} \circ \alpha\right)(t)} .
$$

Furthermore, if $\alpha$ is an integral curve of $\Pi$, then

$$
\frac{d}{d t}\left(\tau_{2}^{1} \circ \alpha\right)=\Pi \circ \alpha
$$

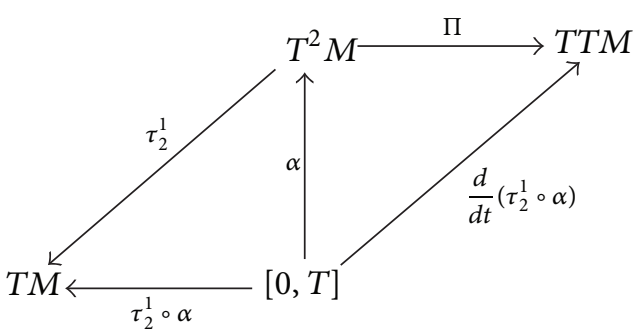


Now, if $\alpha$ is locally represented by $\left(x^{i} ; y^{i} ; u^{i}\right)$, in order to describe the differential equations (23), we should consider (25) with

$$
\Pi^{i} \circ \alpha=y^{i}, \quad \Pi^{i+n} \circ \alpha=u^{i}-\sum_{j, k=1}^{n} \Gamma_{j k}^{i} y^{j} y^{k} .
$$

The variables $\left(x^{i} ; y^{i}\right)$ are called the state variables and $u^{i}$ are the control variables of our control problem.

The optimal control problem consists in finding the curves $\alpha:[0, T] \rightarrow T^{2} M$ of class $C^{2}$, piecewise smooth, with fixed endpoints in the state space

$$
\left(\tau_{2}^{1} \circ \alpha\right)(0)=\left(x_{0}, y_{0}\right), \quad\left(\tau_{2}^{1} \circ \alpha\right)(T)=\left(x_{T}, y_{T}\right),
$$

satisfying the control system $(d / d t)\left(\tau_{2}^{1} \circ \alpha\right)=\Pi \circ \alpha$ and minimizing the functional $\int_{0}^{T} G(\alpha(t)) d t$, for $G: T^{2} M \rightarrow \mathbb{R}$ the cost function defined by

$$
G\left([\gamma]_{0}^{2}\right)=\frac{1}{2}\left\langle K\left(\Pi\left([\gamma]_{0}^{2}\right)\right), K\left(\Pi\left([\gamma]_{0}^{2}\right)\right)\right\rangle
$$

for each $[\gamma]_{0}^{2} \in T^{2} M$, where $K$ is the connection application defined by (21). Notice that in local coordinates, the cost function is given by

$$
G\left(x^{i} ; y^{i} ; u^{i}\right)=\frac{1}{2} \sum_{i, j=1}^{n} g_{i j} u^{i} u^{j}
$$

The relation between (19) and (30) is $L\left(x^{i} ; y^{i} ; u^{i}-\right.$ $\left.\sum_{k=1}^{n} \Gamma_{i j}^{k} y^{i} y^{j}\right)=G\left(x^{i} ; y^{i} ; u^{i}\right)$, or equivalently, $L\left(x^{i} ; y^{i} ; u^{i}\right)=$ $G\left(x^{i} ; y^{i} ; \bar{u}^{i}\right)$.

4.2. Presymplectic Hamiltonian System. The Hamiltonian description of our problem has the cotangent bundle $T^{*} T M$ as costate space. We consider the presymplectic system $(\mathscr{T}$, $\Omega, H)$ characterized as follows.

(i) The total space is the bundle over TM given by

$$
\mathscr{T}=T^{*} T M \times_{T M} T^{2} M
$$

where the fiber of $\mathscr{T}$ over a point $(x, y) \in T M$ is $T_{(x, y)}^{*} T M \times\left(\tau_{2}^{1}\right)^{-1}(x, y)$. The canonical projections are $p r_{1}: T^{*} T M \times_{T M} T^{2} M \rightarrow T^{*} T M$ and $p r_{2}:$ $T^{*} T M \times_{T M} T^{2} M \rightarrow T^{2} M$.

(ii) The presymplectic 2 -form $\Omega$ on $\mathscr{T}$ is defined by the pullback

$$
\Omega=\left(p r_{1}\right)^{*} \omega_{1}
$$

where $\omega_{1}$ denotes the canonical symplectic 2 -form on $T^{*} T M$.

(iii) The Hamiltonian function $H: \mathscr{T} \rightarrow \mathbb{R}$ is defined by

$$
H=\left\langle\left\langle p r_{1}, \Pi \circ p r_{2}\right\rangle\right\rangle-G \circ p r_{2},
$$

where $\Pi$ and $G$ are defined, respectively, by (27) and $(29)$, and $\langle\langle\cdot, \cdot\rangle\rangle$ represents the pairing duality of vectors and covectors on TM.

(iv) The dynamical vector field $X_{H}: \mathscr{T} \rightarrow T(\mathscr{T})$ is the solution of the dynamical system

$$
i_{X_{H}} \Omega=d H
$$

We now apply the geometric algorithm of presymplectic systems to $(\mathscr{T}, \Omega, H)$. We first consider the submanifold $W_{1}$ defined by (13), but adapted to our second-order problem. In this stage, it is important to do a local analysis of the presymplectic structure $\Omega$ defined by (32). If $\left(x^{i}\right)$ is a local coordinate system on $M$ and $\left(x^{i} ; y^{i} ; p_{i} ; q_{i}\right)$ and $\left(x^{i} ; y^{i} ; u^{i}\right)$ represent, respectively, the natural local coordinates on $T^{*} T M$ and $T^{2} M$, then $\left(x^{i} ; y^{i} ; u^{i} ; p_{i} ; q_{i}\right)$ is a coordinate system on the total space $\mathscr{T}=T^{*} T M \times_{T M} T^{2} M$. In this context, it is obvious that $\Omega$ is expressed by

$$
\Omega=\sum_{i=1}^{n}\left(d x^{i} \wedge d p_{i}+d y^{i} \wedge d q_{i}\right)
$$

and so $\operatorname{Ker} \Omega=\operatorname{span}\left\{\partial / \partial u^{1}, \ldots, \partial / \partial u^{n}\right\}$. It follows that $W_{1}$ is locally defined by the constraints

$$
\frac{\partial H}{\partial u^{i}}=0, \quad i=1, \ldots, n .
$$

Note also that since our control system is affine on the controls, from (33) and (30), we get $\left(\partial^{2} H\right) /\left(\partial u^{i} \partial u^{j}\right)=$ $-\left(\partial^{2} G\right) /\left(\partial u^{i} \partial u^{j}\right)=-g_{i j}, 1 \leq i, j \leq n$. As a consequence, the matrix

$$
\left[\frac{\partial^{2} H}{\partial u^{i} \partial u^{j}}\right]_{1 \leq i, j \leq n}
$$

is invertible, and this means that the system is regular at any point.

Consider $\Omega_{W_{1}}$ the restriction to $W_{1}$ of the presymplectic form $\Omega$ defined by (32).

Proposition 1. The 2-form $\Omega_{W_{1}}$ is symplectic.

Proof. Recall that $\Omega_{W_{1}}$ is symplectic if and only if $T_{z} W_{1} \cap$ $\left(T_{z} W_{1}\right)^{\perp}=\{0\}$, for all $z \in W_{1}$, where

$$
\left(T_{z} W_{1}\right)^{\perp}=\left\{v_{z} \in T_{z} \mathscr{T}: \Omega(z)\left(v_{z}, u_{z}\right)=0, \forall u_{z} \in T_{z} W_{1}\right\}
$$

Let $z \in W_{1}$. Using the fact that $W_{1}$ is defined by (36), we can conclude that $\partial / \partial u^{j} \notin T_{z} W_{1}$, for $j=1, \ldots, n$. Indeed, suppose that there exists a $j$ such that $\partial / \partial u^{j} \in T_{z} W_{1}$; this means that $d\left(\partial H / \partial u^{i}\right)\left(\partial / \partial u^{j}\right)=\left(\partial^{2} H\right) /\left(\partial u^{i} \partial u^{j}\right)=0$, for $i=$ $1, \ldots, n$, but this is not true because of the invertibility of the matrix (37). Therefore,

$$
T_{z} W_{1} \oplus \operatorname{Ker} \Omega(z)=T_{z} \mathscr{T}
$$


To conclude the proof, we have just to observe that $\operatorname{Ker} \Omega(z)=$ $\left(T_{z} W_{1}\right)^{\perp}$. It is sufficient to verify that $\left(T_{z} W_{1}\right)^{\perp} \subset \operatorname{Ker} \Omega(z)$ since, by definition, the opposite inclusion always happens. If $v_{z} \in\left(T_{z} W_{1}\right)^{\perp}$, then $\Omega(z)\left(v_{z}, u_{z}\right)=0$, for all $u_{z} \in T_{z} W_{1}$. Furthermore, from $\partial / \partial u^{i} \in \operatorname{Ker} \Omega$, we get $\Omega(z)\left(v_{z}, \partial / \partial u^{i}\right)=$ 0 , for all $i=1, \ldots, n$. In this way, according to (39), we conclude that $v_{z} \in \operatorname{Ker} \Omega(z)$, and hence $\operatorname{Ker} \Omega(z)=\left(T_{z} W_{1}\right)^{\perp}$. Consequently, as we have the direct sum (39), we get $T_{z} W_{1} \cap$ $\left(T_{z} W_{1}\right)^{\perp}=\{0\}$.

The previous proposition assures us that $\left(W_{1}, \Omega_{W_{1}}\right)$ is a symplectic manifold. As a result, the algorithm stops after the first step because we can state that there exists a unique vector field $X_{W_{1}}$ on $W_{1}$ solution of the dynamical system (34) when restricted to $W_{1}$, that is, such that

$$
i_{X_{W_{1}}} \Omega_{W_{1}}=d H_{W_{1}}
$$

where $H_{W_{1}}$ is the restriction of $H$ to $W_{1}$.

We proceed with the analysis of the obtained system and an important simplification of our study. Using (27) and (30), we obtain the following local expression for the Hamiltonian $H: \mathscr{T} \rightarrow \mathbb{R}$ defined by (33):

$$
\begin{aligned}
& H\left(x^{i} ; y^{i} ; u^{i} ; p_{i} ; q_{i}\right) \\
& \quad=\sum_{i=1}^{n} p_{i} y^{i}+\sum_{i=1}^{n} q_{i}\left(u^{i}-\sum_{j, k=1}^{n} \Gamma_{j k}^{i} y^{j} y^{k}\right)-\frac{1}{2} \sum_{i, j=1}^{n} g_{i j} u^{i} u^{j} .
\end{aligned}
$$

Then, the submanifold $W_{1}$ is defined by

$$
q_{i}=\sum_{j=1}^{n} g_{i j} u^{j}, \quad i=1, \ldots, n
$$

and this implies that the optimal controls are

$$
u^{i}=\sum_{j=1}^{n} g^{i j} q_{j}, \quad i=1, \ldots, n .
$$

So, we can consider the diffeomorphism

$$
\begin{gathered}
\varphi:\left(T^{*} T M, \omega_{1}\right) \longrightarrow\left(W_{1}, \Omega_{W_{1}}\right), \\
\left(x^{i} ; y^{i} ; p_{i} ; q_{i}\right) \longmapsto\left(x^{i} ; y^{i} ; \sum_{j=1}^{n} g^{i j} q_{j} ; p_{i}, q_{i}\right) .
\end{gathered}
$$

Observe that the inverse function of $\varphi$ is the restriction to $W_{1}$ of the projection $p r_{1}$. It is easy to show that $\varphi^{*} \Omega_{W_{1}}=\omega_{1}$, which means that $\varphi$ defines a symplectomorphism between the symplectic manifolds $\left(T^{*} T M, \omega_{1}\right)$ and $\left(W_{1}, \Omega_{W_{1}}\right)$. This allows us to reduce the study of the dynamical system (40) on $W_{1}$, to the study of the following system on $T^{*} T M$ :

$$
i_{X_{H_{1}}} \omega_{1}=d H_{1}
$$

where $H_{1}: T^{*} T M \rightarrow \mathbb{R}$ is defined by $H_{1}=H_{W_{1}} \circ \varphi$. Locally,

$$
\begin{aligned}
H_{1} & \left(x^{i} ; y^{i} ; p_{i} ; q_{i}\right) \\
& =\sum_{i=1}^{n} p_{i} y^{i}+\sum_{i=1}^{n} q_{i}\left(\frac{1}{2} \sum_{l=1}^{n} g^{i l} q_{l}-\sum_{j, k=1}^{n} \Gamma_{j k}^{i} y^{j} y^{k}\right)
\end{aligned}
$$

and the vector field $X_{H_{1}}$ on $T^{*} T M$ is the pushforward of $X_{W_{1}}$ by $\varphi^{-1}$; that is,

$$
X_{H_{1}}=\left(\varphi^{-1}\right)_{*} X_{W_{1}}=T \varphi^{-1} \circ X_{W_{1}} \circ \varphi .
$$

The solution vector field is determined according to

$$
\begin{aligned}
& X_{H_{1}}\left(x^{i} ; y^{i} ; p_{i} ; q_{i}\right) \\
& =\sum_{i=1}^{n}\left(\frac{\partial H_{1}}{\partial p_{i}} \frac{\partial}{\partial x^{i}}+\frac{\partial H_{1}}{\partial q_{i}} \frac{\partial}{\partial y^{i}}-\frac{\partial H_{1}}{\partial x^{i}} \frac{\partial}{\partial p_{i}}-\frac{\partial H_{1}}{\partial y^{i}} \frac{\partial}{\partial q_{i}}\right)
\end{aligned}
$$

that is,

$$
\begin{gathered}
X_{H_{1}}\left(x^{i} ; y^{i} ; p_{i} ; q_{i}\right) \\
=\sum_{i=1}^{n}\left[y^{i} \frac{\partial}{\partial x^{i}}+\left(\sum_{j=1}^{n} g^{i j} q_{j}-\sum_{j, k=1}^{n} \Gamma_{j k}^{i} y^{j} y^{k}\right) \frac{\partial}{\partial y^{i}}\right. \\
+\sum_{j, k, l=1}^{n} q_{j}\left(\frac{\partial \Gamma_{l k}^{j}}{\partial x^{i}} y^{l} y^{k}-\frac{1}{2} \frac{\partial g^{j k}}{\partial x^{i}} q_{k}\right) \frac{\partial}{\partial p_{i}} \\
\left.+\left(2 \sum_{j, k=1}^{n} q_{j} \Gamma_{i k}^{j} y^{k}-p_{i}\right) \frac{\partial}{\partial q_{i}}\right]
\end{gathered}
$$

As a consequence, the Hamiltonian equations are

$$
\begin{aligned}
& \dot{x}^{i}=y^{i} \\
& \dot{y}^{i}=\sum_{j=1}^{n} g^{i j} q_{j}-\sum_{j, k=1}^{n} \Gamma_{j k}^{i} y^{j} y^{k}, \\
& \dot{p}_{i}=\sum_{j, k, l=1}^{n} q_{j}\left(\frac{\partial \Gamma_{l k}^{j}}{\partial x^{i}} y^{l} y^{k}-\frac{1}{2} \frac{\partial g^{j k}}{\partial x^{i}} q_{k}\right), \\
& \dot{q}_{i}=2 \sum_{j, k=1}^{n} q_{j} \Gamma_{i k}^{j} y^{k}-p_{i},
\end{aligned}
$$

for $i=1, \ldots, n$.

\section{Examples}

5.1. Optimal Control Problem on the Euclidean Space $\mathbb{R}^{n}$. A trivial example of the optimal control problem discussed in the previous section is the case $M=\mathbb{R}^{n}$. The tangent space of $\mathbb{R}^{n}$ at an arbitrary point can be identified with $\mathbb{R}^{n}$, and the Riemannian metric on $\mathbb{R}^{n}$ is the Euclidean one. By means of 
the canonical basis $\left\{e_{i}\right\}$ of $\mathbb{R}^{n}$, the components of the metric are $g_{i j}=e_{i} \cdot e_{j}=\delta_{i j}$ and the Christoffel symbols are all null.

The cost functional of the control problem is

$$
G: \mathbb{R}^{3 n} \longrightarrow \mathbb{R}, \quad G\left(x^{i} ; y^{i} ; u^{i}\right)=\frac{1}{2} \sum_{i=1}^{n}\left(u^{i}\right)^{2},
$$

and the control system, described by a vector field $\Pi: \mathbb{R}^{3 n} \rightarrow$ $\mathbb{R}^{4 n}$ along the projection $\tau_{2}^{1}: \mathbb{R}^{3 n} \rightarrow \mathbb{R}^{2 n}$, is locally given by

$$
\dot{x}^{i}=y^{i}, \quad \dot{y}^{i}=u^{i}, \quad i=1, \ldots, n .
$$

The Hamiltonian $H: \mathbb{R}^{5 n} \rightarrow \mathbb{R}$ of the presymplectic system that describes the dynamics of the problem is

$$
H\left(x^{i} ; y^{i} ; u^{i} ; p_{i} ; q_{i}\right)=\sum_{i=1}^{n}\left[p_{i} y^{i}+q_{i} u^{i}-\frac{1}{2}\left(u^{i}\right)^{2}\right] \text {. }
$$

Therefore, the constraint equations $\partial H / \partial u^{i}=0, i=1, \ldots, n$, defining the symplectic manifold $W_{1}$ are $u^{i}=q_{i}, i=1, \ldots, n$. Consequently, the Hamiltonian $H_{1}$ defined on the symplectic manifold $\mathbb{R}^{4 n}$ becomes

$$
H_{1}\left(x^{i} ; y^{i} ; p_{i} ; q_{i}\right)=\sum_{i=1}^{n}\left[p_{i} y^{i}+\frac{1}{2}\left(q_{i}\right)^{2}\right] .
$$

From (48), we know that $X_{H_{1}}\left(x^{i} ; y^{i} ; p_{i} ; q_{i}\right)=\sum_{i=1}^{n}\left[y^{i}\left(\partial / \partial x^{i}\right)+\right.$ $\left.q_{i}\left(\partial / \partial y^{i}\right)-p_{i}\left(\partial / \partial q_{i}\right)\right]$ is the solution vector field on $\mathbb{R}^{4 n}$ and the corresponding Hamiltonian equations are

$$
\begin{aligned}
& \dot{x}^{i}=y^{i}, \\
& \dot{y}^{i}=q_{i}, \\
& \dot{p}_{i}=0, \\
& \dot{q}_{i}=-p_{i},
\end{aligned}
$$

for $i=1, \ldots, n$. Note that these equations give the equations $d^{4} x^{i} / d t^{4}=0, i=1, \ldots, n$, and so the curve $\gamma$ in $\mathbb{R}^{n}$ locally represented by $\left(x^{i}\right)$ is such that $d^{4} \gamma / d t^{4}=0$. This corresponds to (16), since, on the Riemannian manifold $\mathbb{R}^{n}$, the covariant derivative along a curve is the usual derivative along a curve in $\mathbb{R}^{n}$ and the curvature tensor is null. We have obtained the equation of cubic polynomials on the Euclidean space as we would expect.

5.2. Optimal Control Problem on the Sphere $S^{2}$. Let $S^{2}=\{x \in$ $\left.\mathbb{R}^{3}:\|x\|=a\right\}$ be the sphere of radius $a$ with the usual spherical coordinates $x^{1}=\theta$ and $x^{2}=\varphi$. $S^{2}$ is equipped with the Riemannian metric $d s^{2}=a^{2} \sin ^{2} \varphi d \theta^{2}+a^{2} d \varphi^{2}$, and so $g_{12}=g_{21}=0, g_{22}=a^{2}$, and $g_{11}=a^{2} \sin ^{2} \varphi$. Using (18), we obtain the Christoffel symbols: $\Gamma_{12}^{1}=\Gamma_{21}^{1}=\cot \varphi$, $\Gamma_{11}^{2}=-\sin \varphi \cos \varphi$, and all the others are null.

Consider the optimal control problem of the previous section on $M=S^{2}$. The state space is $T S^{2}$, the bundle of controls is $T^{2} S^{2}$, and the costate space is $T^{*} T S^{2}$. We represent the corresponding coordinates on these spaces by $\left(\theta, \varphi, y_{\theta}, y_{\varphi}\right)$, $\left(\theta, \varphi, y_{\theta}, y_{\varphi}, u^{1}, u^{2}\right)$, and $\left(\theta, \varphi, y_{\theta}, y_{\varphi}, p_{\theta}, p_{\varphi}, q_{\theta}, q_{\varphi}\right)$, respectively. The cost function of the control problem is

$$
\begin{gathered}
G: T^{2} S^{2} \longrightarrow \mathbb{R}, \\
G\left(\theta, \varphi, y_{\theta}, y_{\varphi}, u^{1}, u^{2}\right)=\frac{1}{2}\left[a^{2}\left(u^{1}\right)^{2} \sin ^{2} \varphi+a^{2}\left(u^{2}\right)^{2}\right],
\end{gathered}
$$

and the control system is represented by

$$
\begin{aligned}
\dot{\theta} & =y_{\theta}, \\
\dot{\varphi} & =y_{\varphi}, \\
\dot{y}_{\theta} & =u^{1}-2 y_{\theta} y_{\varphi} \cot \varphi, \\
\dot{y}_{\varphi} & =u^{2}+\left(y_{\theta}\right)^{2} \sin \varphi \cos \varphi .
\end{aligned}
$$

The Hamiltonian is defined on the presymplectic manifold $T^{*} T S^{2} \times_{T S^{2}} T^{2} S^{2}$ by

$$
\begin{aligned}
H( & \left.\theta, \varphi, y_{\theta}, y_{\varphi}, u^{1}, u^{2}, p_{\theta}, p_{\varphi}, q_{\theta}, q_{\varphi}\right) \\
= & p_{\theta} y_{\theta}+p_{\varphi} y_{\varphi}+q_{\theta}\left(u^{1}-2 y_{\theta} y_{\varphi} \cot \varphi\right) \\
& +q_{\varphi}\left(u^{2}+\left(y_{\theta}\right)^{2} \sin \varphi \cos \varphi\right) \\
& -\frac{1}{2}\left[a^{2}\left(u^{1}\right)^{2} \sin ^{2} \varphi+a^{2}\left(u^{2}\right)^{2}\right]
\end{aligned}
$$

We can verify that the constraint equations $\partial H / \partial u^{i}=0, i=$ 1,2 , which define the symplectic manifold $W_{1}$, are

$$
q_{\theta}-a^{2} u^{1} \sin ^{2} \varphi=0, \quad q_{\varphi}-a^{2} u^{2}=0,
$$

and consequently the controls are given by

$$
u^{1}=\frac{1}{a^{2}} q_{\theta} \operatorname{cosec}^{2} \varphi, \quad u^{2}=\frac{1}{a^{2}} q_{\varphi}
$$

Therefore, the Hamiltonian defined on $T^{*} T S^{2}$ is written as

$$
\begin{aligned}
& H_{1}\left(\theta, \varphi, y_{\theta}, y_{\varphi}, p_{\theta}, p_{\varphi}, q_{\theta}, q_{\varphi}\right) \\
& =p_{\theta} y_{\theta}+p_{\varphi} y_{\varphi}+\frac{1}{2 a^{2}}\left(q_{\theta}\right)^{2} \operatorname{cosec}^{2} \varphi+\frac{1}{2 a^{2}}\left(q_{\varphi}\right)^{2} \\
& \quad+q_{\varphi}\left(y_{\theta}\right)^{2} \sin \varphi \cos \varphi-2 q_{\theta} y_{\theta} y_{\varphi} \cot \varphi .
\end{aligned}
$$

Hence, according to (48), the solution vector field $X_{H_{1}}$ on $T^{*} T S^{2}$ is given by

$$
\begin{gathered}
X_{H_{1}}\left(\theta, \varphi, y_{\theta}, y_{\varphi}, p_{\theta}, p_{\varphi}, q_{\theta}, q_{\varphi}\right) \\
=y_{\theta} \frac{\partial}{\partial \theta}+y_{\varphi} \frac{\partial}{\partial \varphi}
\end{gathered}
$$




$$
\begin{aligned}
& +\left(\frac{1}{a^{2}} q_{\theta} \operatorname{cosec}^{2} \varphi-2 y_{\theta} y_{\varphi} \cot \varphi\right) \frac{\partial}{\partial y_{\theta}} \\
& +\left[\frac{1}{a^{2}} q_{\varphi}+\left(y_{\theta}\right)^{2} \sin \varphi \cos \varphi\right] \frac{\partial}{\partial y_{\varphi}} \\
& +\left[\frac{1}{a^{2}}\left(q_{\theta}\right)^{2} \operatorname{cosec}^{2} \varphi \cot \varphi-2 q_{\theta} y_{\theta} y_{\varphi} \operatorname{cosec}^{2} \varphi\right. \\
& \left.+q_{\varphi}\left(y_{\theta}\right)^{2}+2 q_{\varphi}\left(y_{\theta}\right)^{2} \sin ^{2} \varphi\right] \frac{\partial}{\partial p_{\varphi}} \\
& +\left(2 q_{\theta} y_{\varphi} \cot \varphi-2 q_{\varphi} y_{\theta} \sin \varphi \cos \varphi-p_{\theta}\right) \frac{\partial}{\partial q_{\theta}} \\
& +\left(2 q_{\theta} y_{\theta} \cot \varphi-p_{\varphi}\right) \frac{\partial}{\partial q_{\varphi}} .
\end{aligned}
$$

The Hamiltonian equations are

$$
\begin{aligned}
\dot{\theta}= & y_{\theta}, \\
\dot{\varphi}= & y_{\varphi}, \\
\dot{y}_{\theta}= & \frac{1}{a^{2}} q_{\theta} \operatorname{cosec}^{2} \varphi-2 y_{\theta} y_{\varphi} \cot \varphi, \\
\dot{y}_{\varphi}= & \frac{1}{a^{2}} q_{\varphi}+\left(y_{\theta}\right)^{2} \sin \varphi \cos \varphi, \\
\dot{p}_{\theta}= & 0 \\
\dot{p}_{\varphi}= & \frac{1}{a^{2}}\left(q_{\theta}\right)^{2} \operatorname{cosec}{ }^{2} \varphi \cot \varphi-2 q_{\theta} y_{\theta} y_{\varphi} \operatorname{cosec}^{2} \varphi \\
& -q_{\varphi}\left(y_{\theta}\right)^{2}+2 q_{\varphi}\left(y_{\theta}\right)^{2} \sin ^{2} \varphi, \\
\dot{q}_{\theta}= & 2 q_{\theta} y_{\varphi} \cot \varphi-2 q_{\varphi} y_{\theta} \sin ^{2} \cos \varphi-p_{\theta}, \\
\dot{q}_{\varphi}= & 2 q_{\theta} y_{\theta} \cot \varphi-p_{\varphi} .
\end{aligned}
$$

5.3. Optimal Control Problem on the Hyperbolic Plane $\mathscr{H}^{2}$. Let us analyze now the optimal control problem of the previous section on the upper half-plane model of the hyperbolic plane:

$$
\mathscr{H}^{2}=\left\{x^{1}+i x^{2} \in \mathbb{C}: x^{2}>0\right\} .
$$

The Riemannian metric on $\mathscr{H}^{2}$ is $d s^{2}=\left[d\left(x^{1}\right)^{2}+d\left(x^{2}\right)^{2}\right] /$ $\left(x^{2}\right)^{2}$, and so its components are $g_{11}=g_{22}=1 /\left(x^{2}\right)^{2}$ and $g_{12}=g_{21}=0$. From (18), we get $\Gamma_{12}^{1}=\Gamma_{21}^{1}=\Gamma_{22}^{2}=-\Gamma_{11}^{2}=$ $-1 / x^{2}$ and $\Gamma_{11}^{1}=\Gamma_{22}^{1}=\Gamma_{12}^{2}=\Gamma_{21}^{2}=0$.

In this case, we have the cost function

$$
\begin{gathered}
G: T^{2} \mathscr{H}^{2} \longrightarrow \mathbb{R}, \\
G\left(x^{1}, x^{2}, y^{1}, y^{2}, u^{1}, u^{2}\right)=\frac{1}{2} \frac{\left(u^{1}\right)^{2}+\left(u^{2}\right)^{2}}{\left(x^{2}\right)^{2}}
\end{gathered}
$$

and the control system

$$
\begin{aligned}
& \dot{x}^{1}=y^{1}, \\
& \dot{x}^{2}=y^{2}, \\
& \dot{y}^{1}=u^{1}+2 \frac{y^{1} y^{2}}{x^{2}}, \\
& \dot{y}^{2}=u^{2}+\frac{\left(y^{2}\right)^{2}-\left(y^{1}\right)^{2}}{x^{2}} .
\end{aligned}
$$

The dynamics of the problem is described by a presymplectic system on $T^{*} T \mathscr{H}^{2} \times_{T \mathscr{H}^{2}} T^{2} \mathscr{H}^{2}$ with Hamiltonian defined by

$$
\begin{aligned}
& H\left(x^{1}, x^{2}, y^{1}, y^{2}, u^{1}, u^{2}, p_{1}, p_{2}, q_{1}, q_{2}\right) \\
& =p_{1} y^{1}+p_{2} y^{2}+q_{1}\left(u^{1}+2 \frac{y^{1} y^{2}}{x^{2}}\right) \\
& +q_{2}\left[u^{2}+\frac{\left(y^{2}\right)^{2}-\left(y^{1}\right)^{2}}{x^{2}}\right]-\frac{1}{2} \frac{\left(u^{1}\right)^{2}+\left(u^{2}\right)^{2}}{\left(x^{2}\right)^{2}} .
\end{aligned}
$$

Therefore, the symplectic manifold $W_{1}$ is locally defined by

$$
q_{1}-\frac{u^{1}}{\left(x^{2}\right)^{2}}=0, \quad q_{2}-\frac{u^{2}}{\left(x^{2}\right)^{2}}=0,
$$

and so the controls are such that

$$
u^{1}=q_{1}\left(x^{2}\right)^{2}, \quad u^{2}=q_{2}\left(x^{2}\right)^{2} .
$$

As a consequence, the Hamiltonian $H_{1}$ is defined on $T^{*} T \mathscr{H}^{2}$ by

$$
\begin{aligned}
& H_{1}\left(x^{1}, x^{2}, y^{1}, y^{2}, p_{1}, p_{2}, q_{1}, q_{2}\right) \\
& =p_{1} y^{1}+p_{2} y^{2}+\frac{1}{2}\left(x^{2}\right)^{2}\left(q_{1}\right)^{2}+\frac{1}{2}\left(x^{2}\right)^{2}\left(q_{2}\right)^{2} \\
& +2 q_{1} \frac{y^{1} y^{2}}{x^{2}}+q_{2} \frac{\left(y^{2}\right)^{2}-\left(y^{1}\right)^{2}}{x^{2}} .
\end{aligned}
$$

Moreover, the corresponding Hamiltonian equations are

$$
\begin{aligned}
& \dot{x}^{1}=y^{1}, \\
& \dot{x}^{2}=y^{2} \\
& \dot{y}^{1}=\left(x^{2}\right)^{2} q_{1}+2 \frac{y^{1} y^{2}}{x^{2}} \\
& \dot{y}^{2}=\left(x^{2}\right)^{2} q_{2}+\frac{\left(y^{2}\right)^{2}-\left(y^{1}\right)^{2}}{x^{2}}, \\
& \dot{p}_{1}=0, \\
& \dot{p}_{2}=2 q_{1} \frac{y^{1} y^{2}}{\left(x^{2}\right)^{2}}+q_{2} \frac{\left(y^{2}\right)^{2}-\left(y^{1}\right)^{2}}{\left(x^{2}\right)^{2}}-x^{2}\left(q_{1}\right)^{2}-x^{2}\left(q_{2}\right)^{2},
\end{aligned}
$$




$$
\begin{aligned}
& \dot{q}_{1}=-2 q_{1} \frac{y^{2}}{x^{2}}+2 q_{2} \frac{y^{1}}{x^{2}}-p_{1} \\
& \dot{q}_{2}=-2 q_{1} \frac{y^{1}}{x^{2}}-2 q_{2} \frac{y^{2}}{x^{2}}-p_{2} .
\end{aligned}
$$

\section{Acknowledgments}

The work of L. Abrunheiro was supported in part by FEDER funds through COMPETE-Operational Programme Factors of Competitiveness (Programa Operacional Factores de Competitividade), and by Portuguese funds through the Center for Research and Development in Mathematics and Applications and the Fundação para a Ciência e a Tecnologia (FCT), within project PEst-C/MAT/UI4106/2011 with COMPETE no. FCOMP-01-0124-FEDER-022690. The research of M. Camarinha was partially supported by the Centro de Matemática da Universidade de Coimbra, funded by the European Regional Development Fund through the program COMPETE and by the Portuguese Government through the Fundação para a Ciência e a Tecnologia (FCT) under the project PEst-C/MAT/UI0324/2011. The work of J. ClementeGallardo was partially supported by Grants DGA 24/1, MICINN MTM2012-33575, and UZ2012CIE-06.

\section{References}

[1] R. Skinner and R. Rusk, "Generalized Hamiltonian dynamics. I. Formulation on $T^{*} Q \bigoplus T Q$," Journal of Mathematical Physics, vol. 24, no. 11, pp. 2589-2594, 1983.

[2] M. J. Gotay and J. M. Nester, "Presymplectic Lagrangian systems. I. The constraint algorithm and the equivalence theorem," Annales de l'institut Henri Poincaré (A), vol. 30, no. 2, pp. 129$142,1979$.

[3] P. Crouch and F. S. Leite, "The dynamic interpolation problem: on Riemannian manifolds, Lie groups, and symmetric spaces," Journal of Dynamical and Control Systems, vol. 1, no. 2, pp. 177202, 1995.

[4] L. Noakes, G. Heinzinger, and B. Paden, "Cubic splines on curved spaces," IMA Journal of Mathematical Control and Information, vol. 6, no. 4, pp. 465-473, 1989.

[5] M. Camarinha, P. Crouch, and F. Silva Leite, "Hamiltonian structure of generalized cubic polynomials," in Proceedings of the IFAC Workshop on Lagrangian and Hamiltonian Methods for Nonlinear Control, pp. 13-18, Princeton University, Princeton, NJ, USA, March 2000

[6] M. Barbero-Liñan, A. Echeverría-Enríquez, D. Martín de Diego, M. C. Muñoz-Lecanda, and N. Román-Roy, "SkinnerRusk unified formalism for optimal control systems and applications," Journal of Physics A, vol. 40, no. 40, pp. 12071-12093, 2007.

[7] M. Delgado-Tellez and A. Ibort, "A panorama of geometrical optimal control theory," Extracta Mathematicae, vol. 18, no. 2, pp. 129-151, 2003.

[8] M. de León, J. Cortés, D. Martín de Diego, and S. Martínez, "General symmetries in optimal control," Reports on Mathematical Physics, vol. 53, no. 1, pp. 55-78, 2004.
[9] C. López and E. Martínez, "Sub-Finslerian metric associated to an optimal control system," SIAM Journal on Control and Optimization, vol. 39, no. 3, pp. 798-811, 2000.

[10] L. Abrunheiro, Polinómios cúbicos Riemannianos: abordagem Hamiltoniana e generalizações [Ph.D. thesis], University of Coimbra, Coimbra, Portugal, 2012.

[11] L. Abrunheiro, M. Camarinha, and J. Clemente-Gallardo, "Geometry of a second order optimal control problem. Riemannian cubic polynomials," in Proceedings of the 15th International Fall Workshop on Geometry and Physics, D. Iglesias Ponte, J. C. Marrero González, F. Martín Cabrera, E. Padrón Fernández, and D. Sosa Martín, Eds., vol. 11, pp. 199-204, Canary Islands, Spain, September 2007.

[12] L. Abrunheiro, M. Camarinha, and J. Clemente-Gallardo, "Minimum force optimal control of the spherical free rigid body," in Proceedings of the 9th Portuguese Conference on Automatic Control (Control '10), pp. 333-338, Coimbra, Portugal, September 2010.

[13] L. Abrunheiro, M. Camarinha, and J. Clemente-Gallardo, "Cubic polynomials on Lie groups: reduction of the Hamiltonian system," Journal of Physics A, vol. 44, no. 35, Article ID 355203, 2011.

[14] L. Abrunheiro, M. Camarinha, and J. Clemente-Gallardo, "Reduction of the dynamic optimal control problem of the spherical free rigid body," in Mathematical Papers in Honour of Fátima Silva Leite, Textos de Matemàtica, vol. 43, pp. 1-14, Departamento de Matemàtica da Universidade de Coimbra, Coimbra, Portugal, 2011.

[15] M. de León and P. R. Rodrigues, Generalized Classical Mechanics and Field Theory: A Geometrical Approach of Lagrangian and Hamiltonian Formalisms Involving Higher Order Derivatives, North-Holland Mathematics Studies, vol. 112, North-Holland, Elsiever, Amsterdam, The Netherlands, 1985, edited by L. Nachbin. 


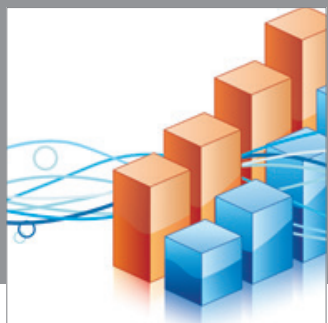

Advances in

Operations Research

mansans

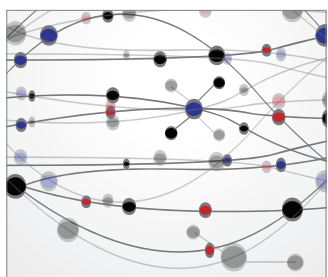

The Scientific World Journal
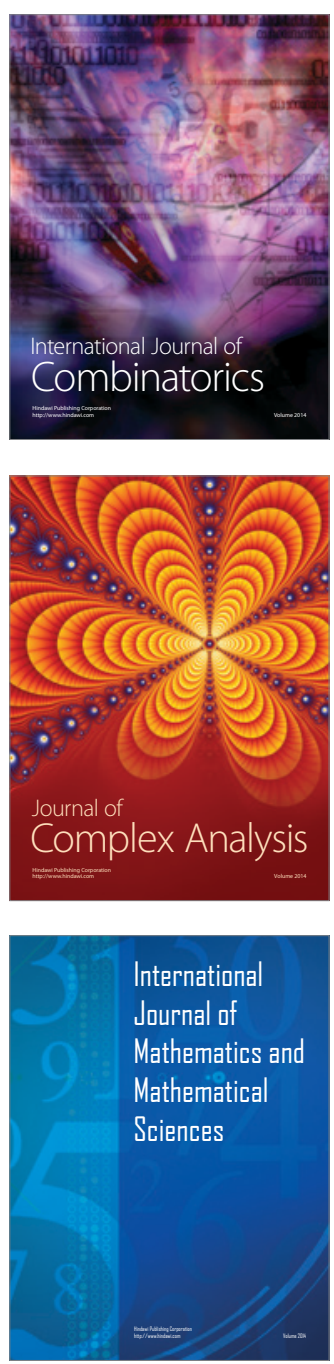
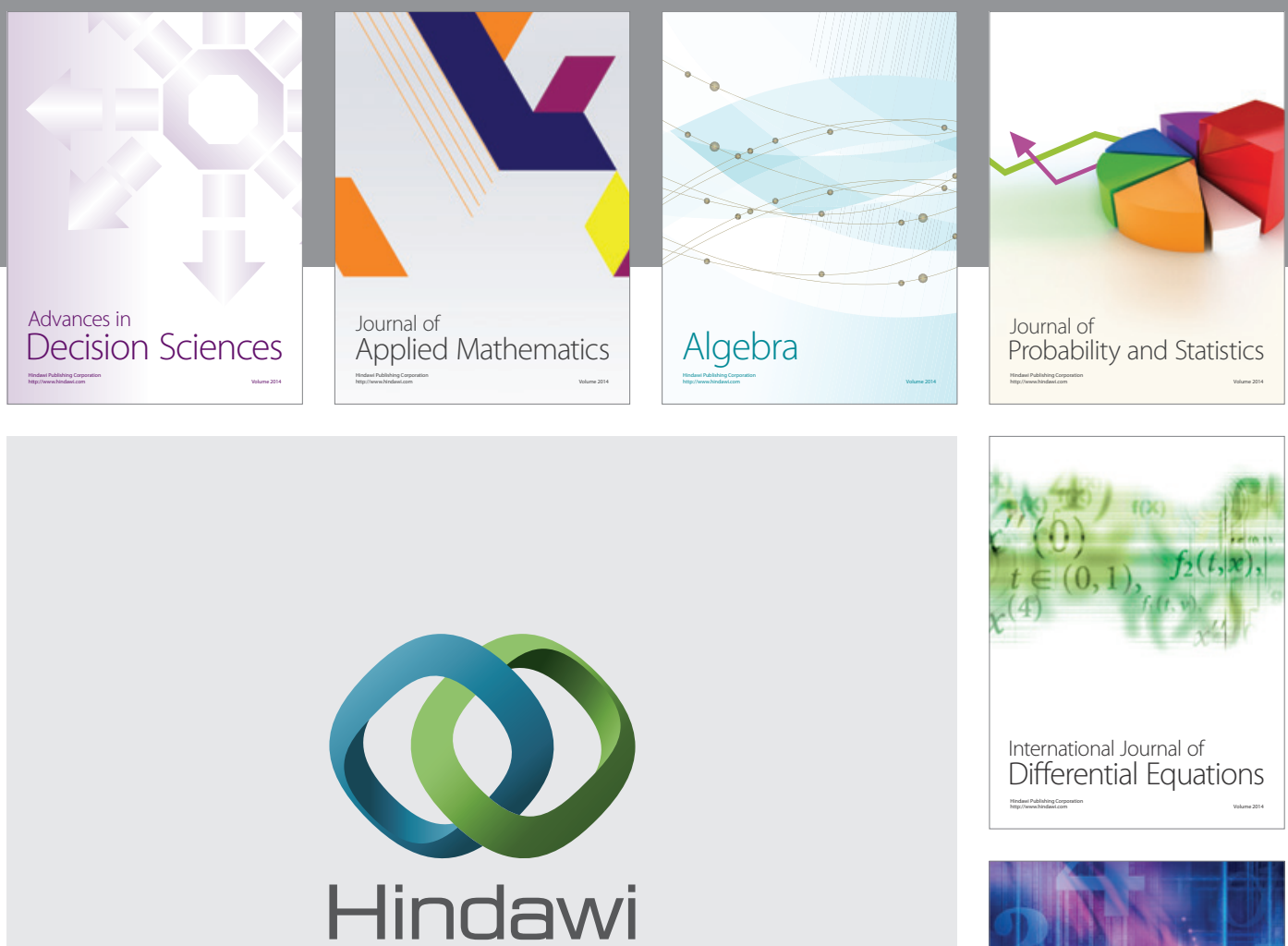

Submit your manuscripts at http://www.hindawi.com
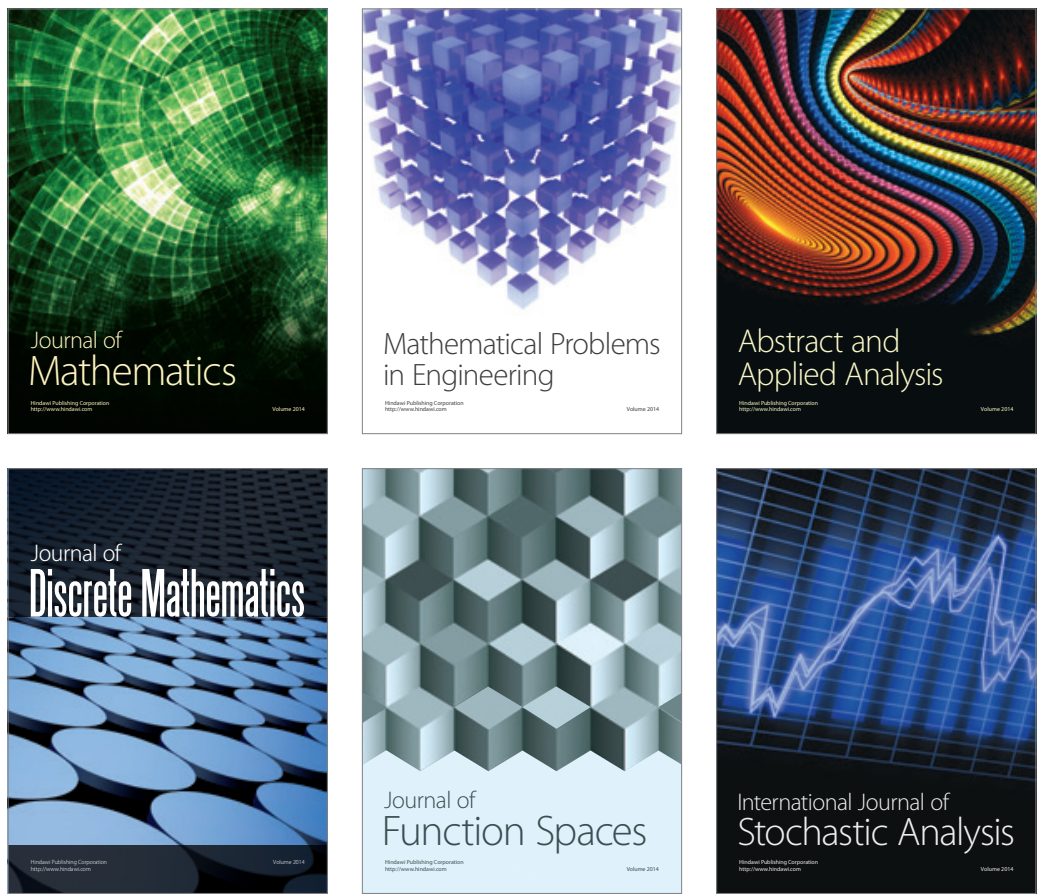

Journal of

Function Spaces

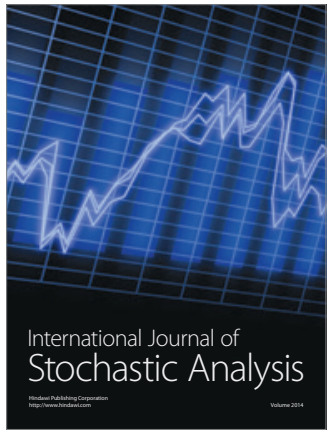

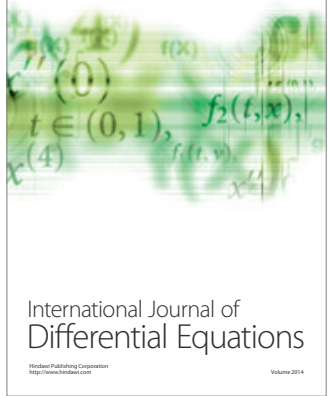
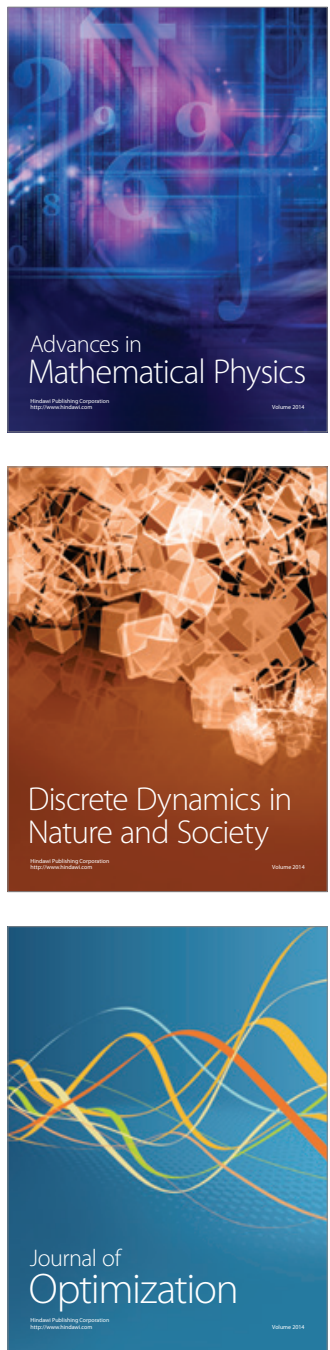